\title{
VLBI OBSERVATIONS OF THE GRAVITATIONAL LENS SYSTEM 2016+112
}

\author{
M. B. Heflin (MIT), M. V. Gorenstein, E. E. Falco, I. I. Shapiro (CfA) \\ B. F. Burke (MIT), J. N. Hewitt, A. E. E. Rogers (Haystack) \\ C. R. Lawrence (CIT)
}

\begin{abstract}
On June 1, 1984 we conducted a seven station 18-cm VLBI observation of the $2016+112$ gravitational lens system. Preliminary brightness distributions for A and B have been obtained via model fitting. Weak correlated flux density was detected in the $\mathrm{C}$ component region.
\end{abstract}

The 2016+112 gravitational lens system has three radio components, labelled A, $B$, and $C$ respectively (1). $A$ and $B$ appear to be gravitationally lensed images of a single source (1). They are both unresolved by the VLA at $6-\mathrm{cm}$ and have identical redshifts of 3.2733 within measurement errors. $C$ is resolved by the VLA at 6-cm. Optical observations suggest that most of the light from the $\mathrm{C}$ component comes from a foreground elliptical galaxy at a redshift of roughly .8 , possibly with some contribution from a weak third image (2).

On June 1, 1984 we conducted a seven station 18-cm VLBI observation of the system. The stations involved were Bonn (B), Green Bank (G), Fort Davis (F), Owens Valley (O), Haystack (K), the VLA (Y), and Arecibo (A). Mark-II recorders were present at all stations. Mark-III recorders were present at all stations except Arecibo. The following analysis involves only the Mark-III data.

Only the $\mathbf{A}$ and $\mathbf{B}$ components were unambiguously detected in fringe rate spectra corresponding to 13 minute integrations. A and B were not detected by the $\mathrm{KF}$ and $\mathrm{KO}$ baselines. There were detection and resolution problems with the F-Y-O triplet data. Our preliminary analysis involves the remaining baselines, which detected and resolved $A$ and $B$.

Standard gain calibration was performed. Plots of correlated flux density suggested two-component circular-gaussian models for the $\mathbf{A}$ and $\mathrm{B}$ images. Residual difference phases were obtained by subtracting an estimate of the geometric difference phase from the observed difference phase. Correlated amplitude, closure phase, and residual difference phase data were used to constrain the $A$ and $\mathrm{B}$ models. The results, which represent a stable chi-squared fit, are illustrated in Figure 1.

VLA observations give values of 56.0 and $61.7 \mathrm{mJy}$ for the $\mathrm{A}$ and $\mathrm{B} 20-\mathrm{cm}$ fluxes respectively (1). Our models yield $S_{A}=36.1 \mathrm{mJy}$ and $S_{B}=38.8 \mathrm{mJy}$, suggesting that extended structures are being overresolved by the baselines included in our analysis. The major discrepancy between the models and the data is found in excess correlated flux of the B data on the K-G baseline near UT $=13$. This excess suggests a jet with a position angle of -95 degrees. Later analysis, including 
the $B$ jet and more short baseline data, may allow matching of $A$ and $B$ features. If such correspondence can be established, calculation of magnification parameters will follow (3). These parameters, which describe a linear mapping of one image into the other, help to constrain models of the lensing mass distribution.

The C component was too weak to be detected unambiguously in individual 13 minute fringe rate spectra. We did, however, detect weak correlated flux density in the $\mathrm{C}$ component region by coherently summing 403 minutes of data from the G-Y baseline, using $B$ as a phase reference (4). The $\mathrm{C}$ component region has a peak signal to noise ratio of 61 . The $\mathrm{B}$ component region has a peak signal to noise of 233 , implying $S_{B} / S_{C}=3.8$ at $18-\mathrm{cm}$.

Future work includes addition of a jet component to the $\mathrm{B}$ model, and incorporation of more short baseline data. Also, VLBI observations at 6-cm will be made in order to detect core-jet morphology at 1 mas resolution. If such structures are found, follow-up observations will search for time dependent source evolution in order to measure the time delay between the $A$ and $B$ images. A well constrained mass distribution for the lens of $2016+112$, along with a time delay between the images, can lead to an estimate of the Hubble constant.
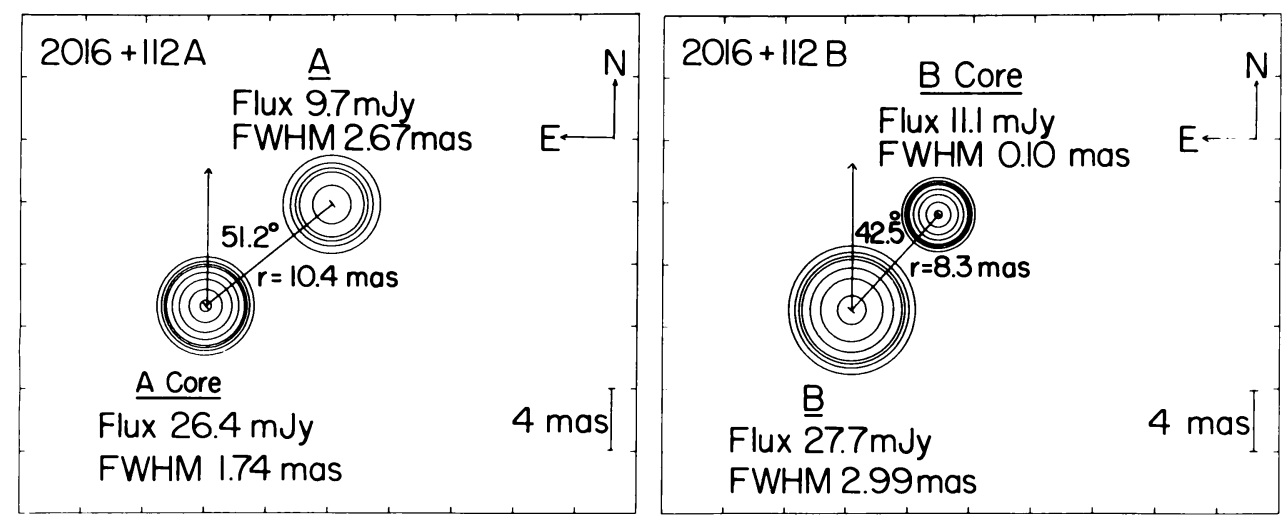

\section{FIGURE 1}

Two component models for $2016+112 \mathrm{~A}$ and B. All of the components are circular gaussians. The more compact component in each model is called the core. The separation A Core - B Core in coordinates of date is $3.02768 " \pm .00006$ " R.A. and $1.5014 " \pm .0003 "$ Dec. The B Core FWHM has an uncertainty of 0.78 mas. All other parameter errors are roughly $5 \%$. These models are based on Mark-III VLBI observations at $18-\mathrm{cm}$.

1. Lawrence, C. R., et al. 1984, Science 223, 46.

2. Schneider, D. P., et al. 1985, Ap. J. 294, 66.

3. Gorenstein, M. V., et al. 1984, Ap. J. 287, 538.

4. Gorenstein, M. V., et al. 1983, Science 219, 54. 